\title{
Bias Tunable Spectral Response of Nanocrystal Array in a Plasmonic Cavity
}

Tung Huu Dang ${ }^{1,2}$, Angela Vasanelli ${ }^{1 *}$, Yanko Todorov ${ }^{1}$, Carlo Sirtori ${ }^{1}$, Yoann Prado ${ }^{2}$, Audrey $\mathrm{Chu}^{2,3}$, Charlie Gréboval ${ }^{2}$, Adrien Khalilii2, Herve Cruguel ${ }^{2}$, Christophe Delerue ${ }^{4}$, Gregory Vincent ${ }^{3}$, Emmanuel Lhuillier ${ }^{2 *}$

${ }^{1}$ Laboratoire de physique de l'Ecole Normale Supérieure, ENS, Université PSL, CNRS, Sorbonne Université, Université de Paris, 75005 Paris, France

2 Sorbonne Université, CNRS, Institut des NanoSciences de Paris, INSP, 75005 Paris, France.

${ }^{3}$ ONERA - The French Aerospace Lab, 6, chemin de la Vauve aux Granges, BP 80100, 91123 Palaiseau, France.

${ }^{4}$ Univ. Lille, CNRS, Centrale Lille, Univ. Polytechnique Hauts-de-France, Junia, UMR 8520 IEMN, F-59000 Lille, France

*To whom correspondence should be sent: angela.vasanelli@ens.fr and el@insp.upmc.fr

\section{Table of content}

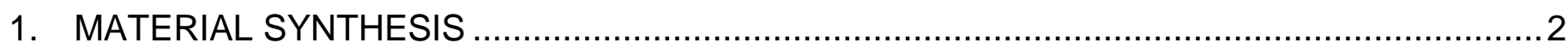

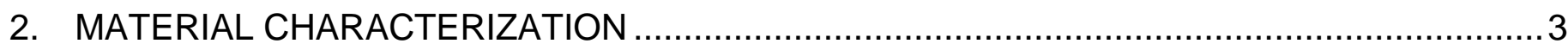

3. ELECTROMAGNETIC SIMULATION AND DESIGN OF THE DEVICE …...........................4

4. ELECTRONIC STRUCTURE UNDER EXTERNAL ELECTRIC FIELD SIMULATION ..............8

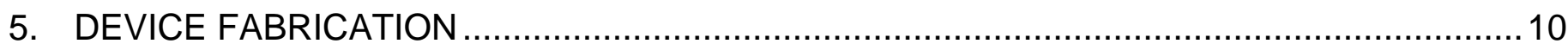

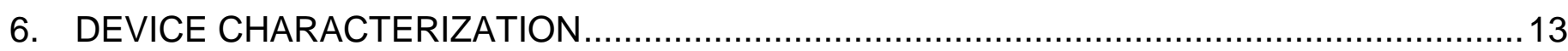

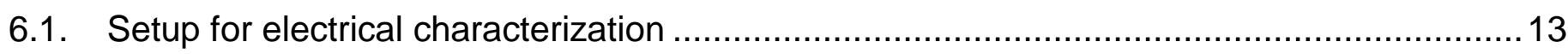

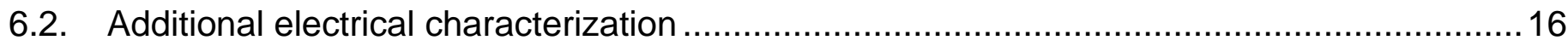

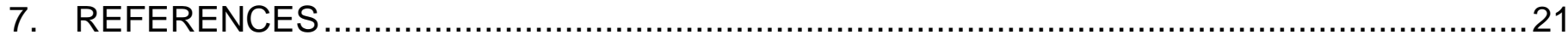




\section{Nanocrystals (NCs) synthesis}

Chemicals: Mercury chloride $\left(\mathrm{HgCl}_{2}\right.$, Sigma-Aldrich, $\left.99 \%\right)$, mercury bromide $\left(\mathrm{HgBr}_{2}\right.$, Alfa Aesar), tellurium powder (Te, Sigma-Aldrich, 99.99\%), trioctylphosphine (TOP, Alfa, 90\%), oleylamine (OLA, Acros, 80-90\%), dodecanethiol (DDT, Sigma-Aldrich, 98\%), 2-mercaptoethanol (MPOH, Merck, $>99 \%$ ), and N,N dimethylformamide (DMF, VWR), toluene (VWR, 99.8\%) were used. All chemicals were used without further purification, except oleylamine that is centrifuged before use. Mercury compounds are highly toxic. Handle them with special care.

$1 \mathrm{M}$ TOP:Te precursor: Te powder $(2.54 \mathrm{~g})$ was mixed in $20 \mathrm{~mL}$ of TOP in a three-neck flask. The flask was kept under vacuum at room temperature for $5 \mathrm{mins}$, then the temperature was raised to $100{ }^{\circ} \mathrm{C}$. Next, degassing of the flask was conducted for the next 20 mins. The atmosphere was switched to nitrogen, and the temperature was raised to $275^{\circ} \mathrm{C}$. The solution was stirred until a clear orange color was obtained. The flask was cooled to room temperature, and the color changed to yellow. Finally, this solution was transferred to a nitrogen-filled glove box for storage.

HgTe NCs growth: In a $50 \mathrm{~mL}$ three-neck flask, $18 \mathrm{~mL}$ of oleylamine was degassed under vacuum and heated to $120^{\circ} \mathrm{C}$ for one hour. Then, the atmosphere was switched to $\mathrm{N}_{2}$, and the temperature was set at $120^{\circ} \mathrm{C}$. Meanwhile, in a vial, $72 \mathrm{mg}$ of $\mathrm{HgBr}_{2}(0.2 \mathrm{mmol})$ was dissolved in $1.8 \mathrm{~mL}$ of OLA under sonication. After degassing, the solution, $0.2 \mathrm{~mL}$ of TOP:Te $(1 \mathrm{M})$, was added $\left(t_{w}=0\right)$. Next, after a waiting time of $t_{w}=10$ mins, the solution was transferred to the syringe, and the content was quickly introduced into hot $\mathrm{OLA}\left(120^{\circ} \mathrm{C}\right)$. The solution color quickly turned dark brown. After 3 mins, a cold mixture (i.e., freezer cooled) of $1 \mathrm{~mL}$ DDT in $2 \mathrm{~mL}$ of toluene was injected, and an ice bath was used to decrease the temperature quickly. The content of the flask was transferred to a centrifuge tube, and QDs were precipitated by adding methanol. After centrifugation, the formed pellet was redispersed in toluene. The solution was precipitated a second time with absolute ethanol and redispersed in toluene. At this step, the QDs were centrifuged in toluene to eliminate the lamellar phase. The solid phase was discarded, and the stable solution phase was transferred to a weighed centrifuge tube and finally precipitated using methanol. Again, the formed pellet (45 mg of dried powder was typically obtained) was redispersed in toluene.

HgTe ink preparation: $10 \mathrm{mg}$ of $\mathrm{HgCl}_{2}, 2 \mathrm{~mL}$ of $\mathrm{MPOH}$, and $18 \mathrm{~mL}$ of DMF are mixed to form an exchange solution. $0.25 \mathrm{~mL}$ of this exchange solution and $0.5 \mathrm{~mL}$ of DMF are added to $0.25 \mathrm{~mL}$ of $\mathrm{HgTe}$ NCs in toluene. Phase dissociation occurs when a few drops of hexane are added to the mixture: the dark bottom phase is where the NCs migrate while the top transparent phase can be removed with a plastic pipette. This washing step is repeated twice before a few $\mathrm{mL}$ of toluene is added, and the mixture is centrifuged at $6000 \mathrm{rpm}$ for 4 minutes. The supernatant is discarded while the formed solid pellets of NCs can be re-dispersed in $100 \mu \mathrm{L}$ of DMF to obtain the ink of HgTe. A few cycles of sonication - vortex - centrifugation can help to promote particle dispersion. 


\section{MATERIAL CHARACTERIZATION}

Infrared Absorption spectroscopy: absorbance spectra of NC solutions and NC inks are taken with a Fisher IS50 Fourier transform infrared spectrometer. In the attenuated total reflection (ATR) mode, a small amount of NC solution (or NC ink) is dropped on the diamond cell and let to dry. The absorbance spectra are acquired over the range from $7500 \mathrm{~cm}^{-1}$ to $500 \mathrm{~cm}^{-1}$ with the resolution of 4 $\mathrm{cm}^{-1}$, after averaging over 32 scans.

Figure $\mathbf{S} 1$ a shows absorbance spectra for NCs in toluene (before ligand exchange, blue line) and NC ink (after ligand exchange, red line). The spectra present a clear absorption peak around 2.5 $\mu \mathrm{m}$ associated with excitons confined in the NCs, and a second peak around $2900 \mathrm{~cm}^{-1}$, due to the $\mathrm{C}-\mathrm{H}$ vibration mode. By comparing the two spectra, it is clear that the ratio of intensity between $\mathrm{C}$ $\mathrm{H}$ mode peak and the exciton peak decreases from NCs in toluene to the ink, indicating an efficient ligand exchange process.

Reflectivity measurements are conducted using a Brucker Vertex 70v Fourier transform Infrared spectrometer. We use the spectrometer in reflection configuration under ambient atmosphere and at room temperature to measure the cavity reflectivity. The source is a Tungsten lamp, and the detector is a DLaTGS. A CaF2 beam-splitter is used. Spectra are typically acquired with a $4 \mathrm{~cm}^{-1}$ resolution and averaged over 32 spectra at least. The reference is made with a gold mirror on the backside of a sapphire substrate.

For TEM imaging, a drop of diluted NC solution was cast on a copper grid covered with an amorphous carbon film. The grid was degassed overnight under secondary vacuum. A JEOL 2010F system was operated at $200 \mathrm{kV}$ for the acquisition of images. Figure $\mathbf{S} \mathbf{1 b}-\mathrm{c}$ provides high-resolution TEM images of sphere-shaped HgTe NCs.

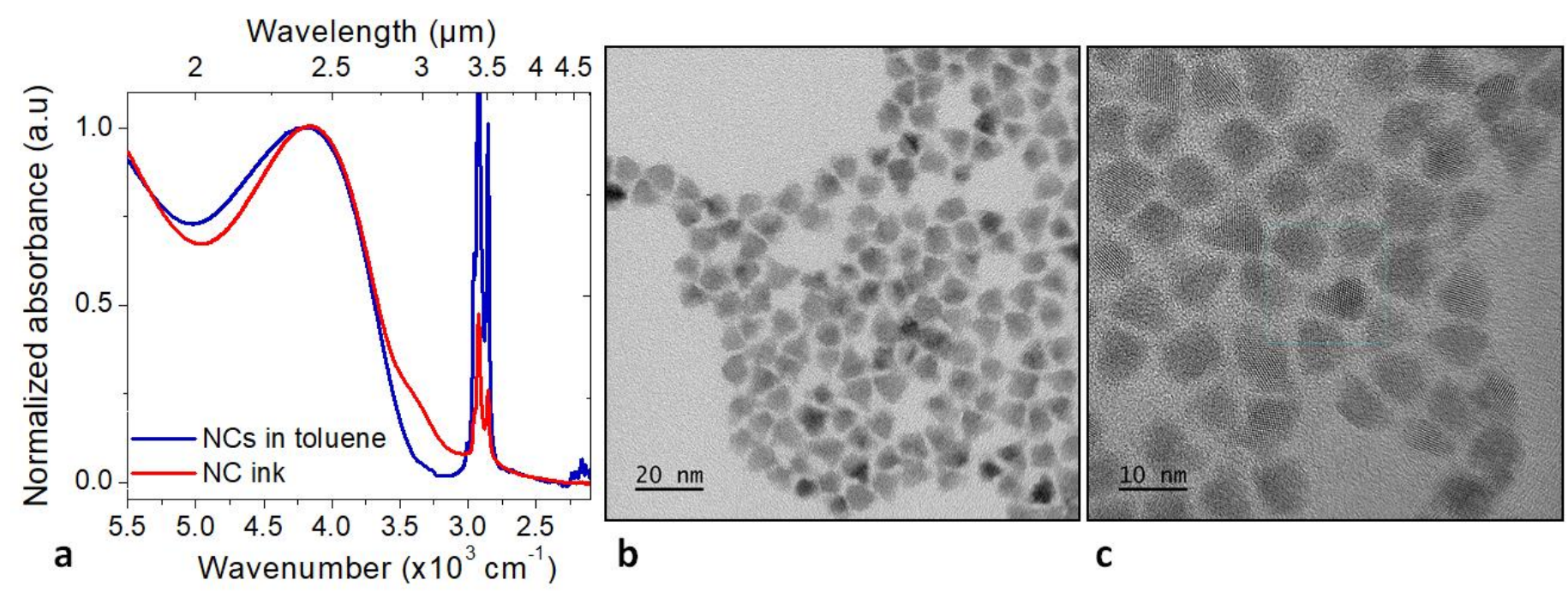

Figure S 1: HgTe nanocrystals: a. Absorbance spectra of NCs in toluene and NC ink (after ligand exchange). b. TEM images of sphere-shaped HgTe NCs. c. High-resolution TEM images of sphereshaped HgTe NCs. 


\section{ELECTROMAGNETIC SIMULATION AND DESIGN OF THE DEVICE}

We use Comsol software to model the propagation of light in our plasmonic system. The structure is simulated in 2D, Frequency Domain Interface. Figure S 2a represents model's geometry for the arrays of plasmonic resonators on a sapphire substrate (see DEVICE FABRICATION paragraph for more details on the selected geometry). The model is constructed for one unit-cell of the resonator array, with Floquet boundary conditions describing the periodicity. In the sapphire layer, a port condition is used for defining the incident wave. On top of the sapphire, we define a perfectly matched layer to absorb all outgoing waves and prevent nonphysical reflections. The power density per unit volume dissipated in the $\mathrm{NC}$ film and metals is calculated from $P=-0.5 \omega|F|^{2} \operatorname{Im}(\varepsilon)$, where $F$ is the electric field, $\omega$ is the angular frequency of the incident wave, and $\operatorname{Im}(\varepsilon)$ is the imaginary part of material permittivity. This quantity, divided by the incident power, gives the absorption per unit volume in the structure.
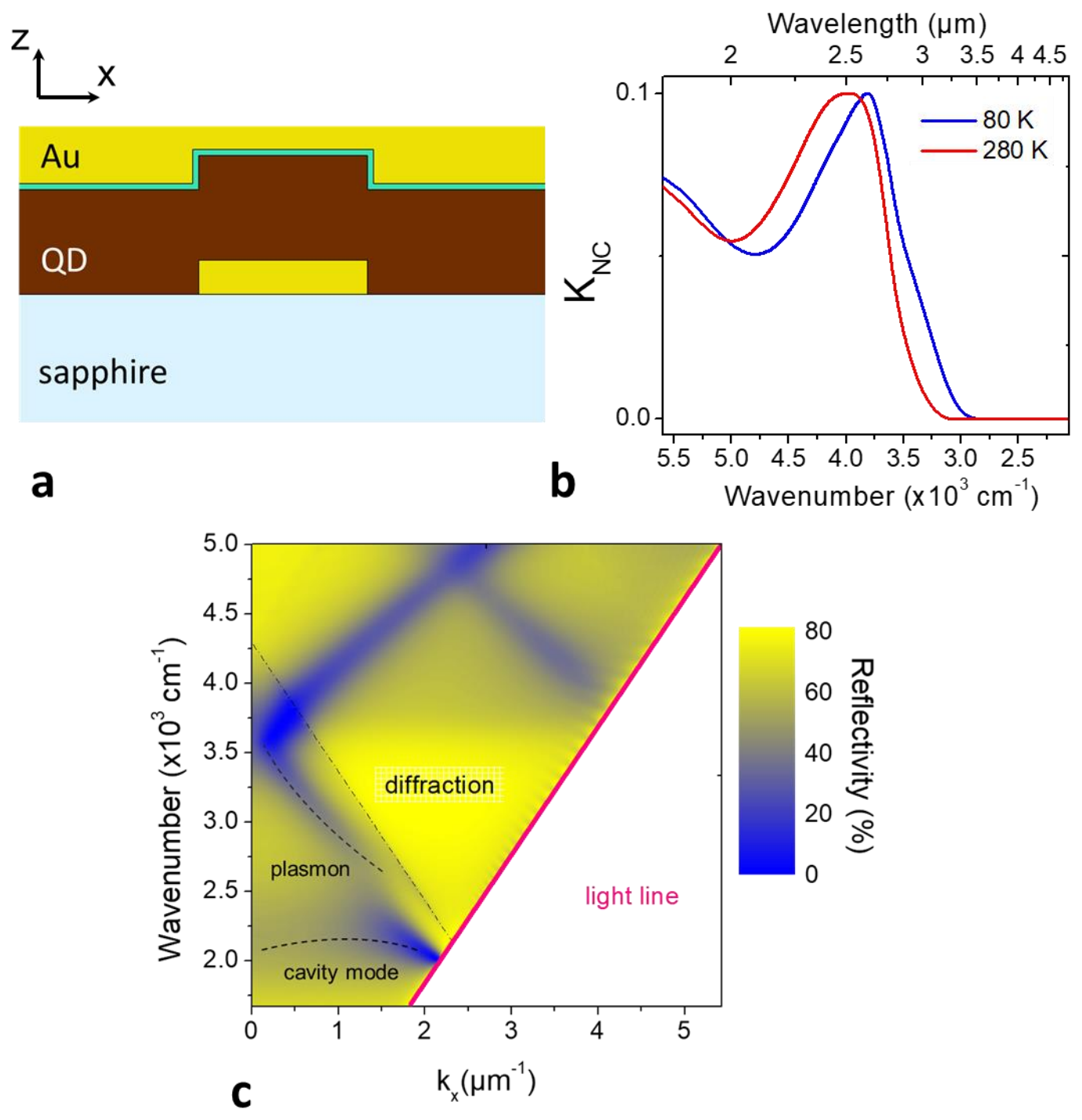

Figure S 2: Design of the cavity: a. Schematic of the cavity. From bottom to top: sapphire substrate - Au stripe - NC layer - PMMA layer (in aqua blue color) - Au mirror. b. NC extinction coefficient spectra estimated from photocurrent spectra of interdigitated sample at $80 \mathrm{~K}$ and $280 \mathrm{~K}$. c. Simulated reflectivity as a function of incident light wavenumber and in-plane wavevector $k_{x}$ for the plasmonic cavity, in TM polarization. 
The first input needed for the model are the optical indices of the materials. For the sapphire substrate and PMMA layer, the real parts of the refractive indices are set to 1.73 and 1.45, respectively, and the imaginary parts are both set to 0 . For gold, the dielectric function from Drude model $\varepsilon(E)=1-2.29 \times 10^{7 /}[E(E+i 130)]$, with $E$ the photon energy in meV, is used. This expression of the dielectric function of gold is slightly modified with respect to Ref ${ }^{1}$ in order to take into account the losses due to the NC-metal interface and the presence of the $\mathrm{Cr}$ adhesion layer. For the NC film, the real part of the refractive index is set to 2.35. The imaginary part of the refractive index for the $\mathrm{NC}$ film, $\operatorname{KNC}(\lambda)$, is estimated from the photocurrent spectrum in the following way. From Beer's law, we have $\operatorname{KCQD}(\lambda) \propto \lambda \cdot \operatorname{Abs}(\lambda)$, with $\operatorname{Abs}(\lambda)$ the absorbance spectrum. The last is proportional to photocurrent spectrum $\operatorname{ph}(\lambda)$ in the vicinity of the exciton peak. The extinction coefficient $\operatorname{kNc}(\lambda)$ of the NC film is thus estimated by normalizing $\lambda . p h(\lambda)$, with the exciton peak set to $0.1 .^{2}$ The spectra for $\operatorname{KNC}(\lambda)$ at $80 \mathrm{~K}$ and $280 \mathrm{~K}$ are shown in Figure $\mathbf{S} 2 \mathrm{~b}$. $\mathrm{KNC}(\lambda, \mathrm{T}=80 \mathrm{~K})$ is used to model the photocurrent spectra which is measured at low temperature $(50 \mathrm{~K}$ to $80 \mathrm{~K})$, while the $\operatorname{KNc}(\lambda, T=280$ $\mathrm{K})$ is used for modeling the reflectivity experiment at room temperature. It is noted that in the reflectivity experiment (see DEVICE CHARACTERIZATION), the beam impinges on our device from the backside (from air to sapphire to the cavity) and with an external angle of incidence of $15^{\circ}$. Thus, it is necessary to consider the refraction of light when passing from air to sapphire with Snell's law. In our model, since the port generating incoming light is located in the sapphire layer, we set the internal angle of incidence to $8.3^{\circ}$.

- Effect of the cavity on the spectral response
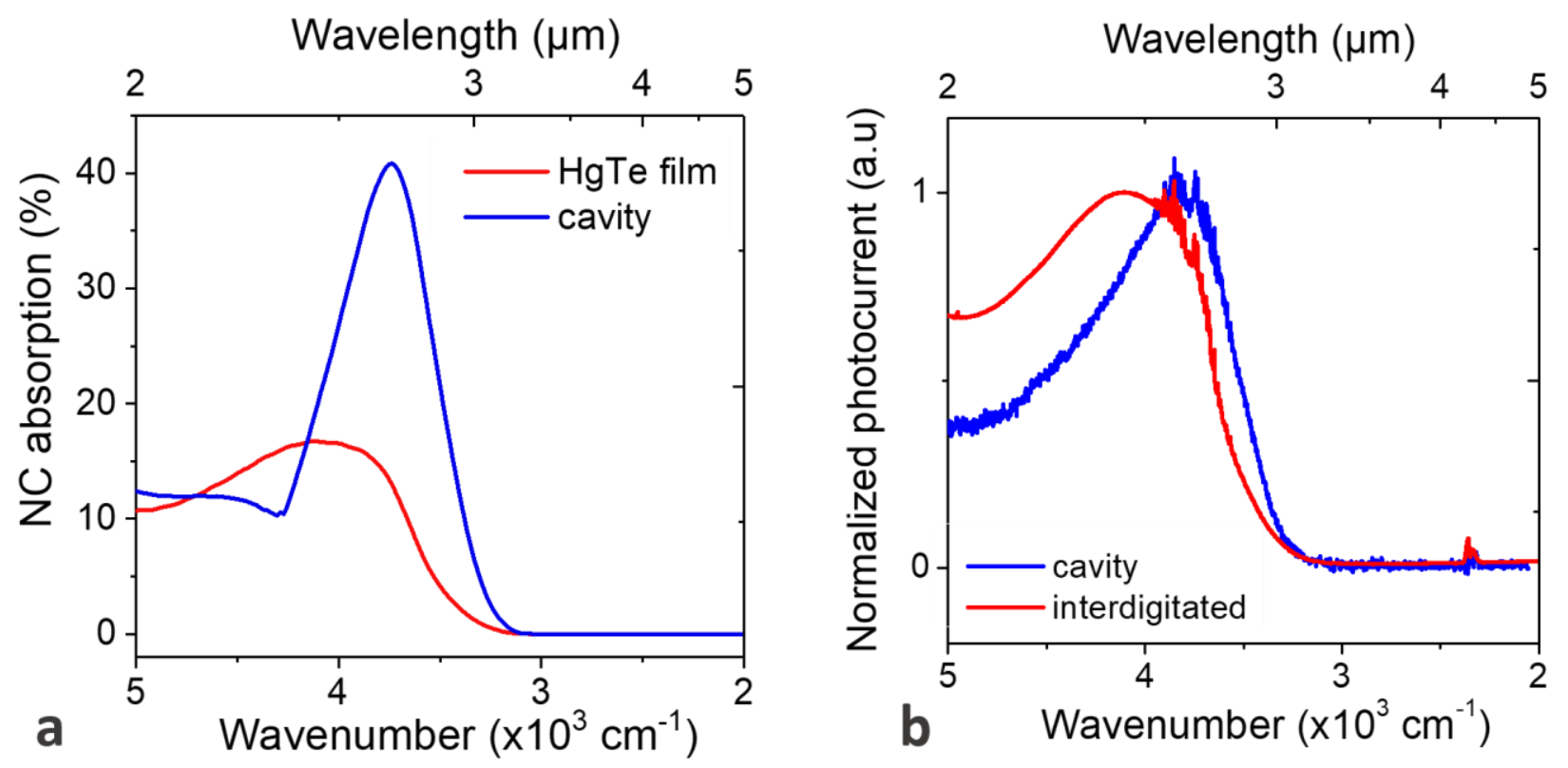

Figure S 3: Effect of the cavity of the device spectrum: a. Simulated NC absorption of a bare $N C$ film (red) and in the presence of the cavity (blue) at normal incidence for TM polarization. $b$. Effect of the cavity on the measured spectral photoresponse. 
- Origin of the bias induced shift in TE polarization

TE $2.8 \mu \mathrm{m}-43 \% \mathrm{NC}$ absorption
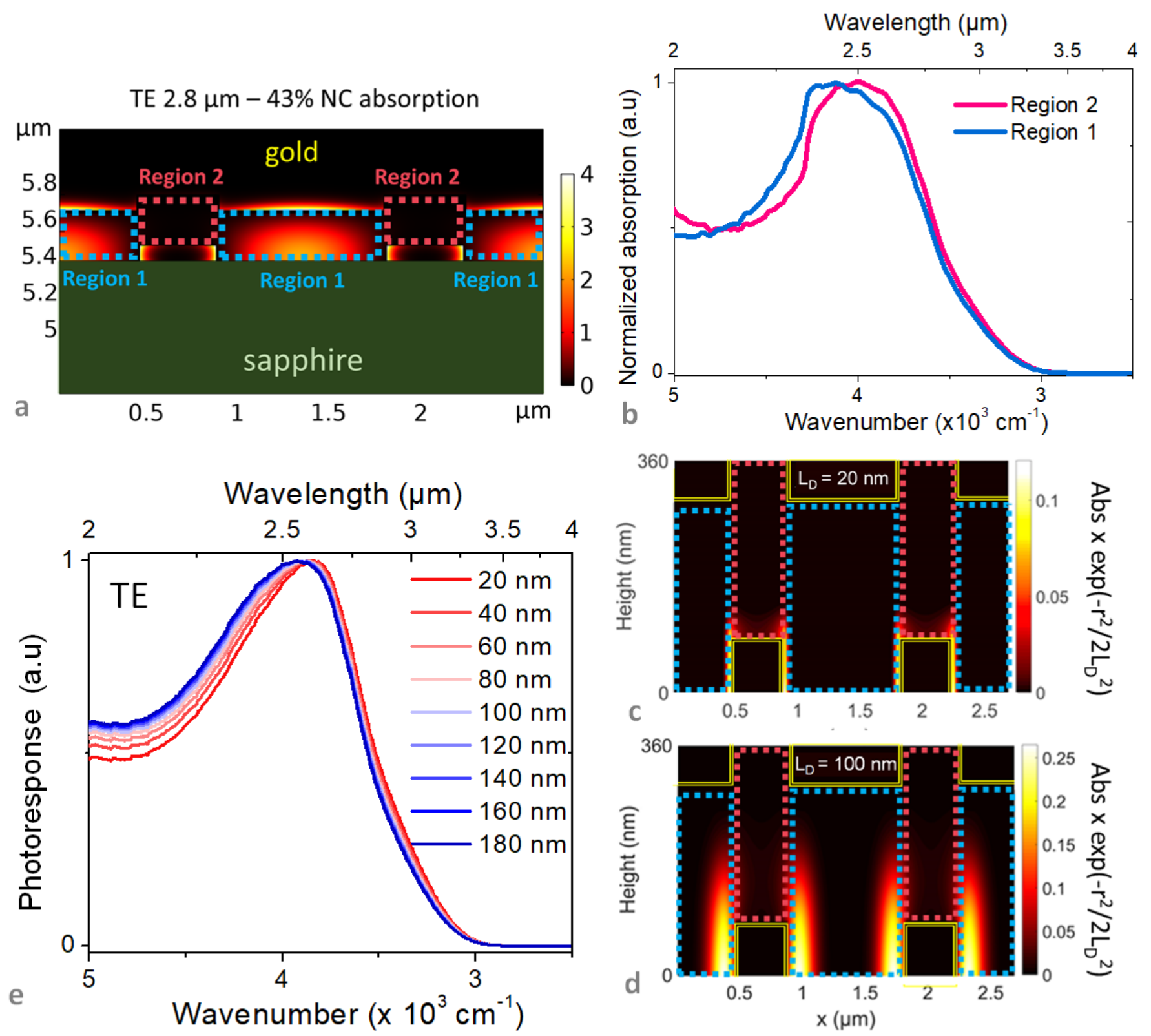

Figure S 4: Origin of the bias induced shift along TE polarization. a. Simulated absorption map in TE polarization for the device depicted in Figure 1a. We define two zones: region 1 is between electrodes, while region 2 is above electrodes. $b$. Simulated absorption spectra in region 1 and 2 defined on part a. c. (resp. d.) product of the absorbance by $\exp \left(-r^{2} /\left(2 L_{D^{2}}\right)\right)$, with $r$ the distance to the electrode while $L_{D}$ is set equal to $20 \mathrm{~nm}$ (resp. $100 \mathrm{~nm}$ ). e. Spectrum of the product of the NC absorbance by $\exp \left(-r^{2} /\left(2 L_{D^{2}}\right)\right)$ for various $L_{D}$ values. Data for TM mode are given in figure 4 of the main text. 


\section{- Effect of the geometrical parameters of the resonator on the spectroscopic properties of the device}

Figure $\mathbf{S} 5$ unveils how grating period (p) and electrode size (s) affect the spectrum of the cavity device. In Figure S 5a, we show how the change of $s$ impacts the spectrum. Mostly the energy of the spoof plasmon is unchanged while the cavity mode blueshifts as $s$ is reduced. Over this broad range of $s$ values, the cavity mode is redder than the exciton. In Figure $\mathbf{S} \mathbf{b} b$, we set the $s$ value equal to $400 \mathrm{~nm}$ and tune the grating period $\mathrm{p}$. A smaller $\mathrm{p}$ leads to a blue shift of the spoof plasmon resonance. In the main text, we focus on a geometry for which the plasmon resonance spectrally overlaps with the exciton. The associated simulated photoresponse (spectrum of the product of absorbance and $\exp \left(-r^{2} /\left(2 L_{D^{2}}\right)\right)$ (Figure $\mathbf{S} 5 \mathrm{~d}$ ) shows a shift of the band edge while the diffusion length is tuned (through bias application). On the other hand, when $p$ is chosen to unmatch plasmon and exciton energies, the simulated photoresponse shows no shift at the band edge while we tune the diffusion length (Figure S 5c).
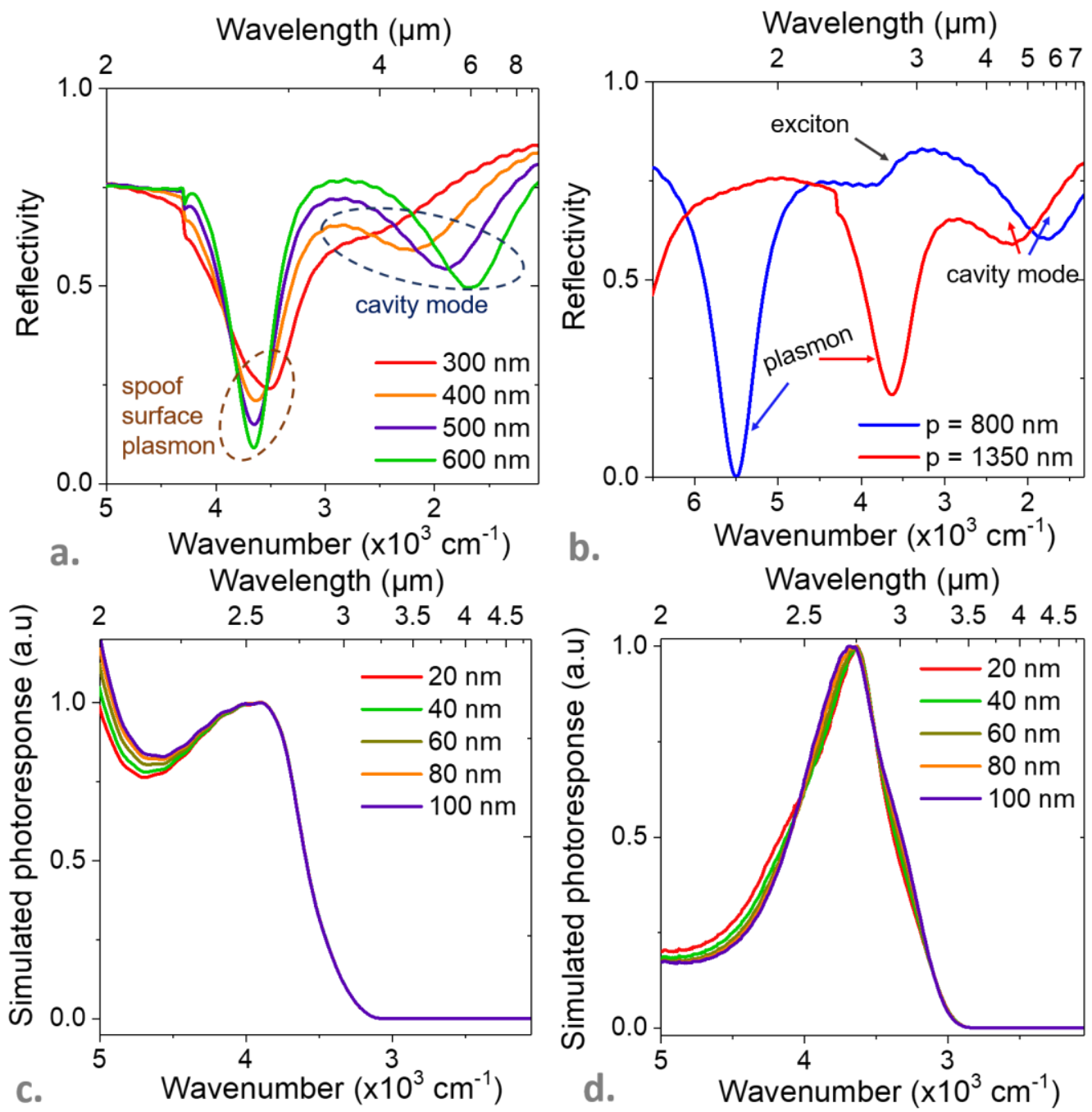

Figure S 5: Effect of the cavity geometrical parameters on its spectroscopic properties. a. Simulated reflectivity spectrum (under normal incidence) when the period $p$ is set to $1350 \mathrm{~nm}$ and the electrode size (s) is tuned. b Simulated reflectivity spectrum (under normal incidence) when the period $p$ is tuned and the electrode size $s$ is fixed to $400 \mathrm{~nm}$. c. (resp. d.) Spectrum of the product of absorbance and $\exp \left(-r^{2} /\left(2 L_{D}^{2}\right)\right)$ for various $L_{D}$ values for a grating period of $800 \mathrm{~nm}$ - no plasmonexciton overlap (resp. $1350 \mathrm{~nm}$ - plasmon overlaps with exciton) while the electrode size $s$ is fixed to $400 \mathrm{~nm}$. 


\section{ELECTRONIC STRUCTURE UNDER EXTERNAL ELECTRIC FIELD SIMULATION}

Tight binding simulation: We used the tight-binding model of Ref 3 to calculate the electronic structure of the HgTe NCs. Each $\mathrm{Hg}$ or Te atoms are described by a double set of $\mathrm{sp}^{3} \mathrm{~d}^{5} \mathrm{~s}^{*}$ orbitals, one for each spin orientation. Surfaces are saturated by pseudo-hydrogen atoms characterized by a single s orbital. Tight-binding parameters, i.e., on-site energies, nearest-neighbor hopping matrix elements, and spin-orbit coupling terms, were determined to provide a very good description of the band structure of bulk HgTe (at $300 \mathrm{~K}$ ). For all NCs, we calculated 60 (1200) conduction (valence) states, and we computed dipolar matrix elements between them as described in Ref 3 . We have assumed a spherical shape for the particle with a diameter of $6.4 \mathrm{~nm}$ corresponding to 4090 atoms. For the simulations under electric field, the field is assumed to be uniform and along the vertical direction.

Figure $\mathbf{S}$ 6a-b shows the absorption cross-section of sphere-shaped HgTe NCs as a function of the applied electric field. The spectra show a clear red-shift trend when a stronger electric field is applied. Under a large field $\left(>100 \mathrm{kV} . \mathrm{cm}^{-1}\right)$, the two states of the first exciton transition start to split, and two distinct contributions can be seen. Normalized first exciton peaks under various electric fields are shown in Figure S $6 \mathrm{~b}$. Besides, wavefunctions of electron (resp. hole) under applied electric field of $10 \mathrm{kV} . \mathrm{cm}^{-1}$ and $200 \mathrm{kV} . \mathrm{cm}^{-1}$ are presented in Figure S $6 \mathrm{c}-\mathrm{d}$ (resp. Figure S $6 \mathrm{e}-\mathrm{f}$ ). It is apparent that the hole wavefunctions are more localized than the electron's one due to strong effective mass differences of holes and electrons in $\mathrm{HgTe} \mathrm{NC}\left(\mathrm{m}^{*} \mathrm{~h} \approx 25 \mathrm{~m}^{*} \mathrm{e}\right)^{4}$. As a result, the hole wavefunction is also more affected by applying an electric field than the electron wavefunction.

The main result of the tight-binding electronic structure simulation is that the Stark effect on such confined HgTe NCs leads to a conventional redshift of the spectrum and is thus not responsible for the observed blueshift of our device. 

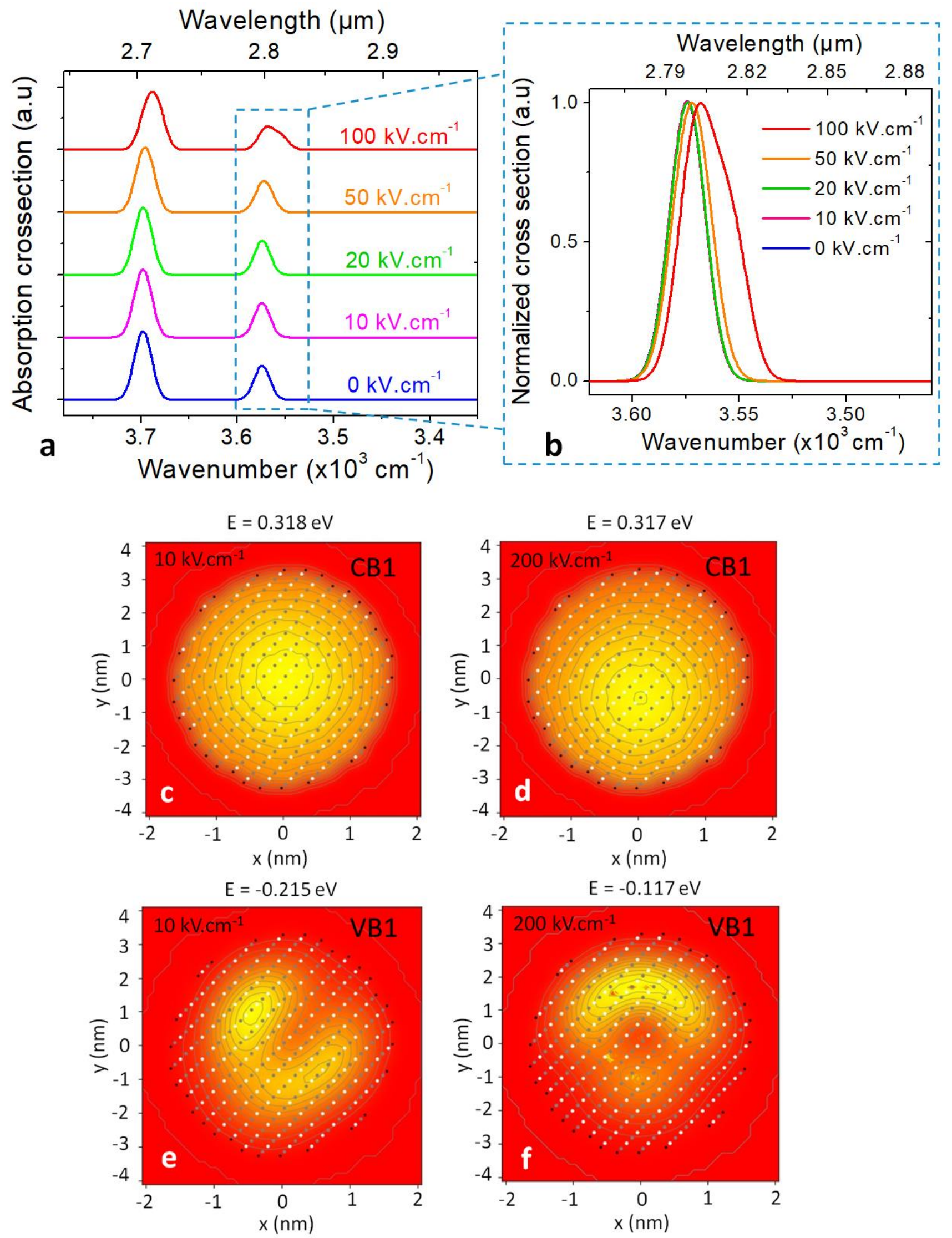

Figure S 6: Tight binding simulation under static electric field. a. Absorption cross-section under different applied electric field. $b$. Normalized absorption cross-section of the 1 st exciton transition. c-d. Electron wavefunction in a sphere-shaped NC under $10 \mathrm{kV} . \mathrm{cm}^{-1}$ and $200 \mathrm{kV} . \mathrm{cm}^{-1}$ 
electric field, respectively. e-f. Hole wavefunction in a sphere-shaped NC under $10 \mathrm{kV} . \mathrm{cm}^{-1}$ and 200 $\mathrm{kV} . \mathrm{cm}^{-1}$ electric field, respectively.

\section{DEVICE FABRICATION}

Figure $\mathbf{S} \mathbf{7}$ describes the fabrication process for our device. In general, the process contains four main stages: optical lithography to fabricate the electrical contacts (Figure S 7a-b-c), e-beam lithography to fabricate the nano-stripes (Figure S $\mathbf{7 d - e}$ ), NC film and PMMA layer deposition (Figure S $\mathbf{7 f}-\mathrm{g}$ ), and evaporation of the top gold mirror and clean up (Figure S $7 \mathrm{~h}-\mathrm{i}$ ). The detailed parameters for each fabrication step are given in the following paragraphs.

\section{a - Sapphire substrate}

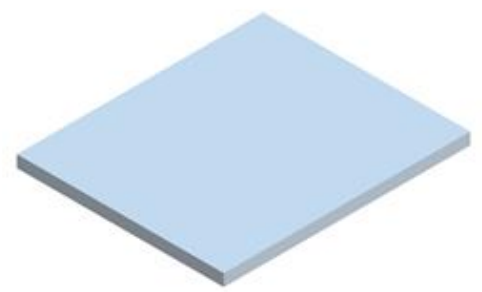

f - $300 \mathrm{~nm}$ HgTe film

by spin-coating

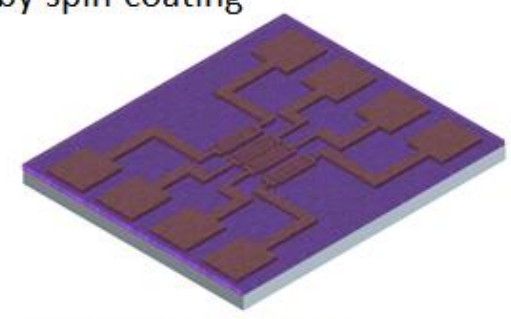

g- 20 nm PMMA
by spin-coating

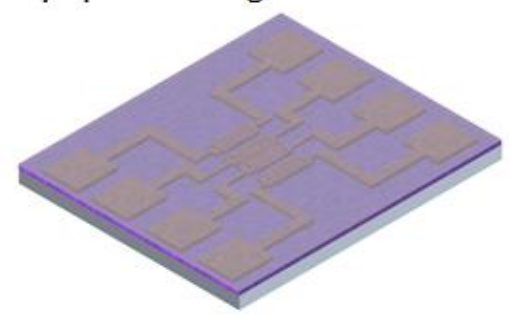

b - AZ5214E photoresist

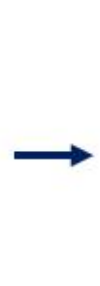

e - $5 \mathrm{~nm} \mathrm{Cr} / 50 \mathrm{~nm}$ Au by

thermal evaporation
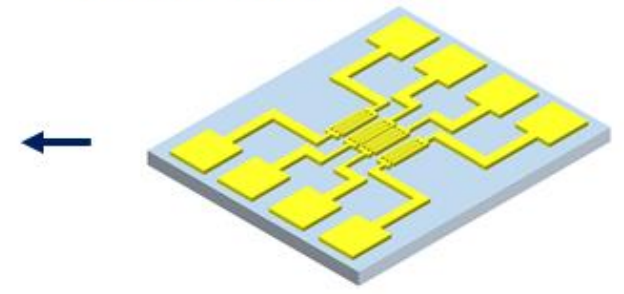

h - $40 \mathrm{~nm}$ Au by

thermal evaporation

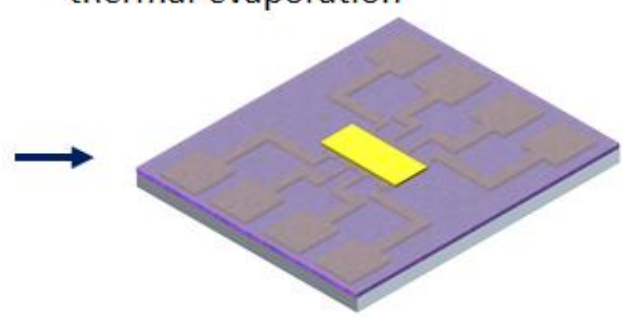

c - $5 \mathrm{~nm} \mathrm{Cr} / 50 \mathrm{~nm}$ Au by

thermal evaporation
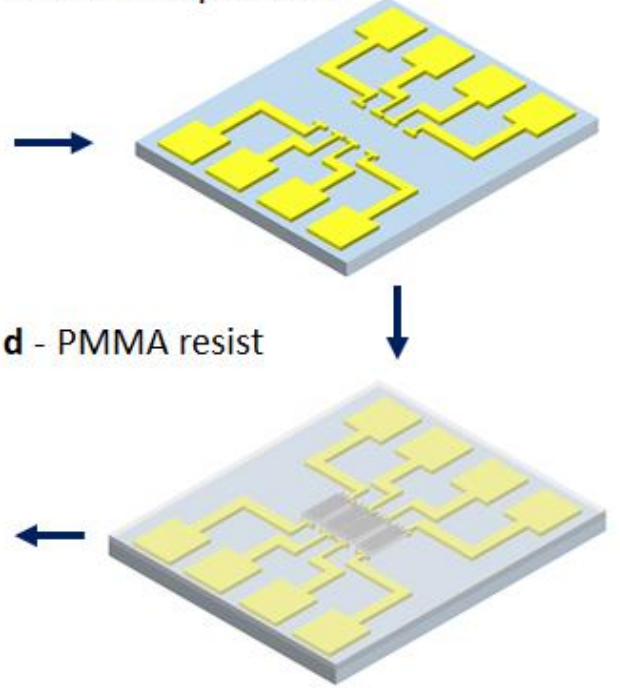

i - Clean up with

a cotton swab

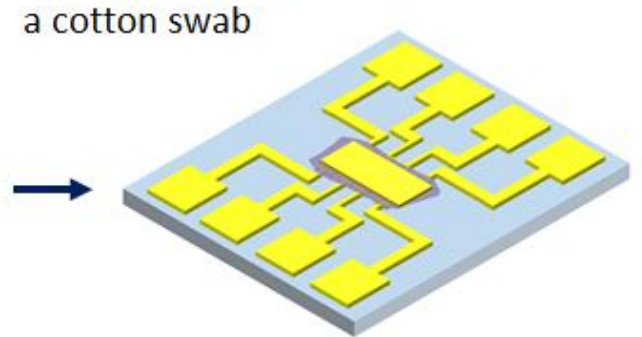

Figure S 7: Fabrication steps for the photodetector

Electrodes for electrical contacts: A sapphire substrate is initially rinsed and sonicated for 5 minutes in acetone to remove dust and organic contaminants. The wafer is then rinsed with isopropanol before being dried with a $\mathrm{N}_{2}$ blow. An additional $\mathrm{O}_{2}$ plasma cleaning step is performed to ensure organic contaminant removal. The TI Prime adhesion promoter is spin-coated onto the sapphire substrate and baked on a hot plate at $120^{\circ} \mathrm{C}$ for 2 minutes. Subsequently, the substrate is spin-coated with AZ5214E photoresist and baked at $110^{\circ} \mathrm{C}$ for 90 seconds. The first UV exposure through a mask is 1.5 seconds, followed by the image reversal bake step at $126{ }^{\circ} \mathrm{C}$ in 2 minutes. A flood exposure for 40 seconds without the mask is then performed. The resist film is developed in AZ726 with a development time of 25 seconds, followed by DI water rinse and $\mathrm{N}_{2}$ gun dry steps. 
The next step is to deposit $5 \mathrm{~nm}$ of $\mathrm{Cr}$ and $50 \mathrm{~nm}$ of Au on the substrate with a thermal evaporator. Afterward, the substrate is soaked in acetone for 3 hours for lift-off.

Fabrication of gold nano-stripes: We employ a standard Raith eLine e-beam lithography system for the fabrication of the nano-stripes. To begin with, $150 \mathrm{~nm}$ PMMA resist is spin-coated onto the sapphire substrate patterned with macroscopic electrodes. The resist is then baked at $175^{\circ} \mathrm{C}$ for 3 minutes. Because sapphire is an insulating material, a layer of $17 \mathrm{~nm}$ of $\mathrm{Al}$ is thermally evaporated on top of the resist to reduce the charging effect. The operating bias of the electron beam is set to $20 \mathrm{kV}$, and the aperture is set to $10 \mu \mathrm{m}$. The dose is set to $280 \mu \mathrm{C} / \mathrm{cm}^{2}$. After the exposure, the Al layer is removed by dipping the film for $60 \mathrm{~s}$ within a diluted solution of $\mathrm{KOH}$. The resist is then developed in a solution of MIBK:isopropanol (1:3 in volume) for 70 seconds and rinsed with isopropanol and then exposed to RIE-ICP oxygen plasma for 5 seconds to eliminate the possible residual resist. $5 \mathrm{~nm} \mathrm{Cr} / 50 \mathrm{~nm} \mathrm{Au}$ is then deposited with a thermal evaporator. The substrate is then dipped in acetone overnight for lift-off. Figure S 8a provides an SEM image for the overview of the grating after the e-beam lithography step.
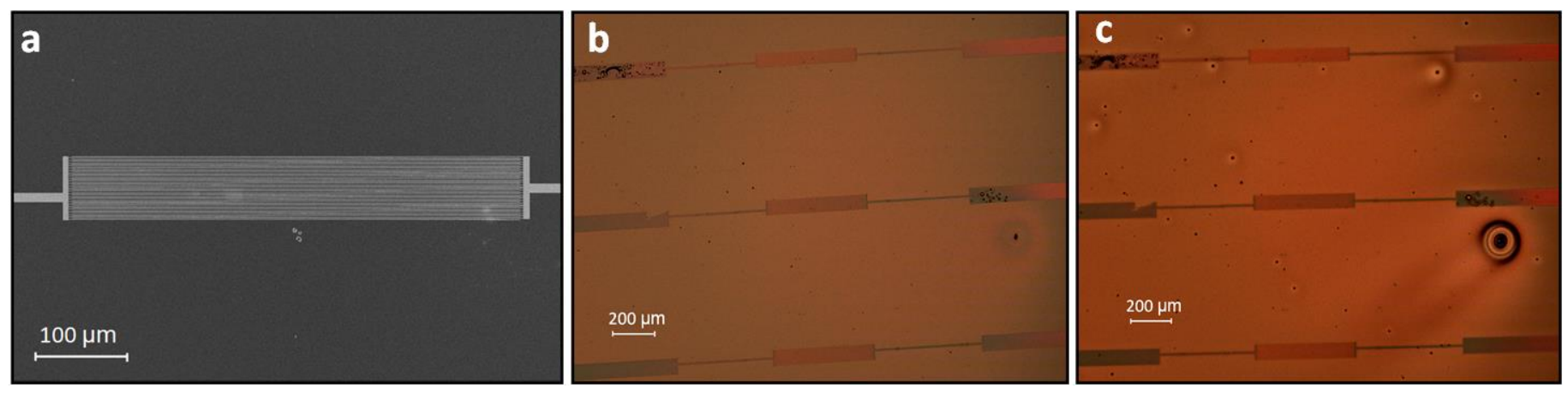

Figure S 8: Images of the device. a. SEM image of the grating after e-beam lithography. $b$. Optical microscopy image of the device after NC deposition. c. Optical microscopy image of the device after NC and PMMA deposition.

NC film and PMMA deposition: A few drops of DMF are spread on the surface of the patterned sapphire substrate before a spin-coating at $3000 \mathrm{rpm}$ for $30 \mathrm{~s}$. This step is necessary to promote the adhesion between the sapphire surface and the NC ink. For a $15 \times 15 \mathrm{~mm}^{2}$ sapphire substrate, 20 $\mu \mathrm{L}$ of $\mathrm{HgTe} \mathrm{NC}$ ink is enough to cover the surface. We use a two-step spin recipe: $1000 \mathrm{rpm}$ for 120 $\mathrm{s}$ followed by $2500 \mathrm{rpm}$ for $30 \mathrm{~s}$. A smooth film of HgTe NCs is formed with a thickness of around $300 \mathrm{~nm}$. By changing spin-coating parameters, it is possible to tune the thickness of the NC films from a few tens of nanometers up to $500 \mathrm{~nm}$. An optical microscopy image of the device after NC deposition is given in Figure $\mathbf{S} \mathbf{8 b}$.

For the PMMA layer, we spin-coat solution of PMMA in anisole/toluene $\left(20 \mathrm{mg} \cdot \mathrm{mL}^{-1}\right)$ on top of the $\mathrm{NC}$ film at a speed of $2000 \mathrm{rpm}$ for $30 \mathrm{~s}$. The thickness of this PMMA layer is about $20 \mathrm{~nm}$. Figure S 8c presents the optical microscopy image of the device after PMMA deposition.

Deposition of the top gold layer: The top gold layer with a thickness of $40 \mathrm{~nm}$ is deposited with a thermal evaporator. A shadow mask is utilized such that this gold layer only covers the area of nanostripes and the NC film. The unwanted areas of NCs are then removed with a cotton swab. This final step completes our resonators in which the $\mathrm{HgTe}$ film plays the role of the active material.

We present in Figure S 9a-b the dark-field microscopy image and the AFM scan image of the complete device, respectively. It is noted that the surface on the grating area is not flat but instead forms a periodic profile, see Figure S $9 \mathrm{c}$. The fluctuations are around $15 \mathrm{~nm}$, which is smaller than 
the thickness of the nano-stripes $(55 \mathrm{~nm})$, implying a compensation of roughness due to the presence of the NC film, the PMMA, and the gold mirror. These fluctuations, however, suggest that the NC and PMMA layers are quasi-conformal and that the real geometry of the cavity is similar to our schematic presented in figure S2a.

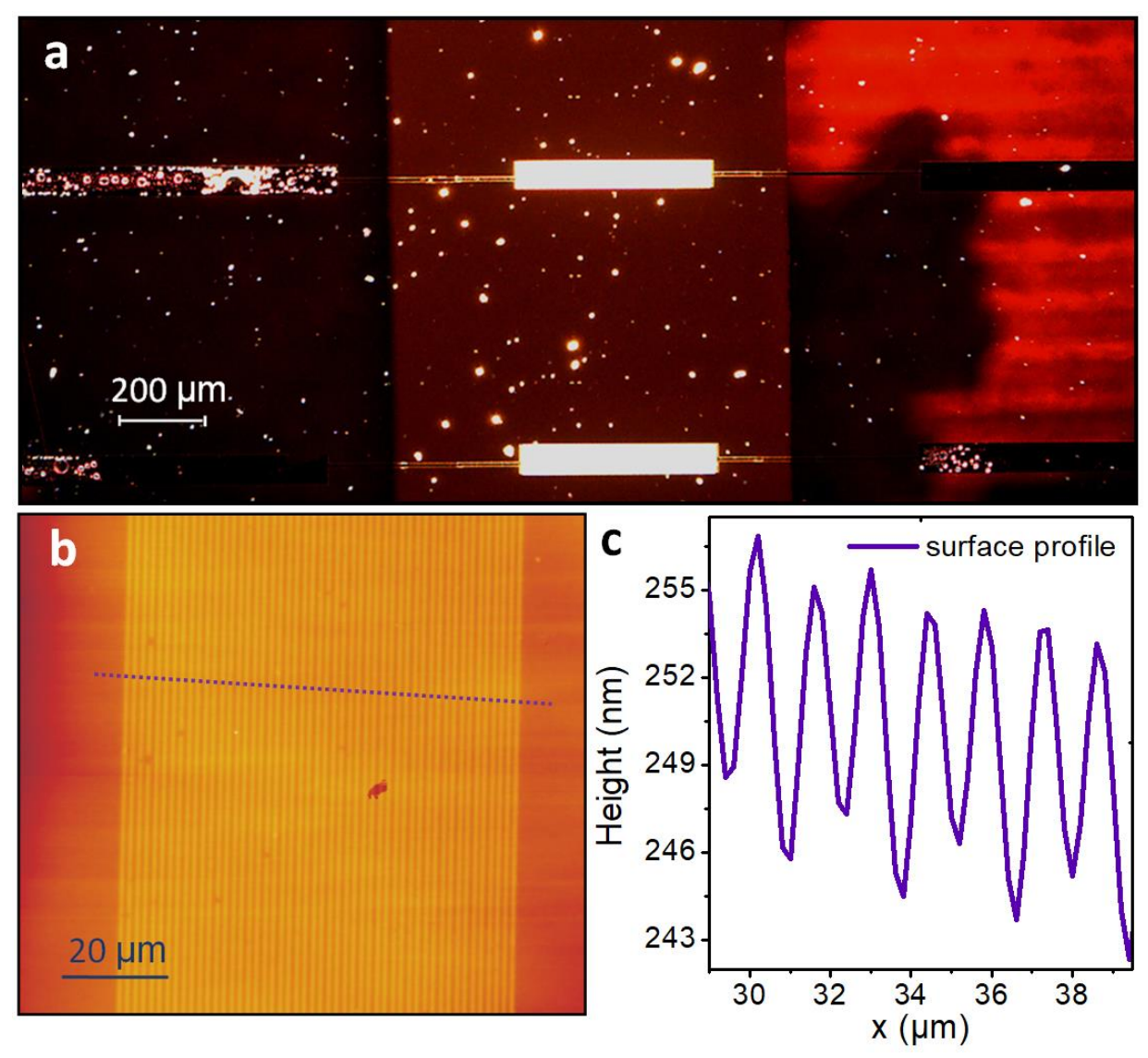

Figure S 9: Topography of the device. a. Dark-field microscopy image of the device after gold mirror deposition. b. AFM topography image of the device after gold mirror deposition. $c$. Surface profile of the purple line in part $b$. 


\section{DEVICE CHARACTERIZATION}

\subsection{Setup for electrical characterization}

Device in the cryostat and electrical powering: The device is mounted on the cold finger of a $\mathrm{He}$ close cycle cryostat. A temperature controller (Lake Shore 335) is used to control and verify the sample temperature. Depending on the measurement, either a Keithley multimeter (Keithley 2634B) or a low noise trans-impedance amplifier (Femto DLPCA-200) can be used as the bias source for the device.

Spectral response at different bias voltages and temperatures: The set-up for measuring photocurrent spectra is illustrated in Figure $\mathbf{S} \mathbf{1 0}$. Our photodetector is illuminated by the Globar beam of the Fourier Transform Infrared spectrometer (FTIR). The device is placed inside the cryostat and biased by a Femto DLPCA 200 amplifier, which is also used for amplifying the photocurrent signal before sending the signal back into the FTIR for spectral analysis. The spectra are typically obtained in continuous scan mode with $4 \mathrm{~cm}^{-1}$ resolution and averaged at last 32 times.

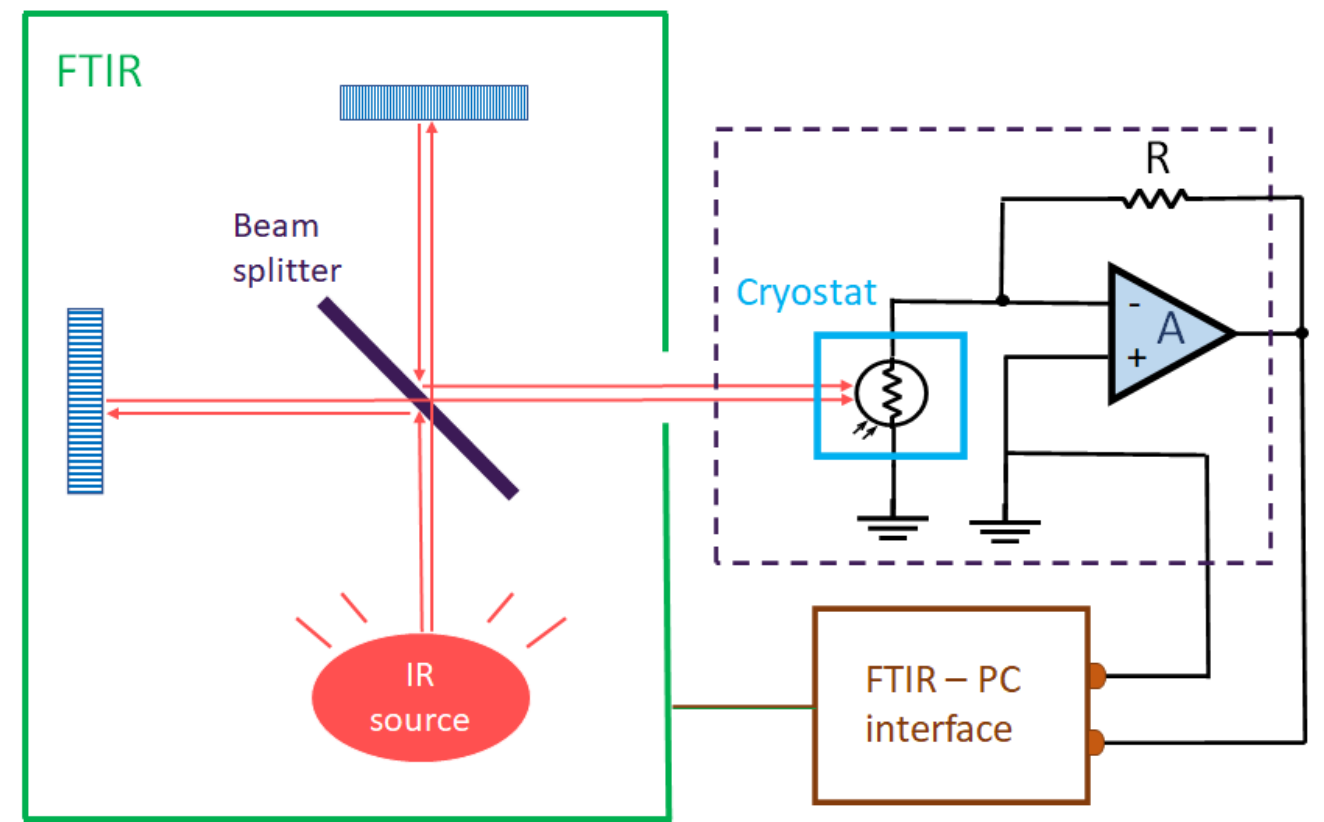

Figure S 10: Spectral photoresponse setup. Experimental set-up for measurements of photocurrent spectra.

Responsivity under blackbody radiation: Figure $\mathbf{S} 11$ illustrates the set-up for responsivity measurement with the blackbody. The device is placed inside the cryostat. A Femto DLPCA 200 amplifier is utilized for biasing the photodetector, acquiring and amplifying the photocurrent signal. The amplified signal is then provided to an oscilloscope. The source is a Blackbody Omega BB-4A set at $980^{\circ} \mathrm{C}$ and placed $24 \mathrm{~cm}$ away from the sample. The incoming flux is chopped by an optical chopper with a frequency varied from $1 \mathrm{~Hz}$ to $1000 \mathrm{~Hz}$. In front of the sample, a Ge window is used to filter out radiation at wavelengths shorter than $1.9 \mu \mathrm{m}$. Thus, the incident power to the sample can be computed using the formula: 


$$
P(W)=\pi A_{d e t} \cdot \sin ^{2} \theta / 2 \cdot \cos \varphi \cdot \int_{1.9 \mu m}^{\lambda_{\text {cut }- \text { off }}} \frac{2 h c^{2}}{\lambda^{5}} \frac{1}{\exp \left(\frac{h c}{\lambda k T}\right)-1} d \lambda
$$

where $A_{\text {det }}$ is the area of the photodetector, $\theta$ is the field of view, $\varphi$ is the incident angle (typically assumed to be $0^{\circ}$ ), $\mathrm{h}$ is the Planck constant, $\mathrm{c}$ is the speed of light, $\mathrm{k}$ is Boltzmann constant, $\mathrm{T}$ is the blackbody temperature equal to $980^{\circ} \mathrm{C}$. $\lambda_{\text {cut-off }}$ is taken from the absorption edge of the material, i.e., $4 \mu \mathrm{m}$ for our NCs.

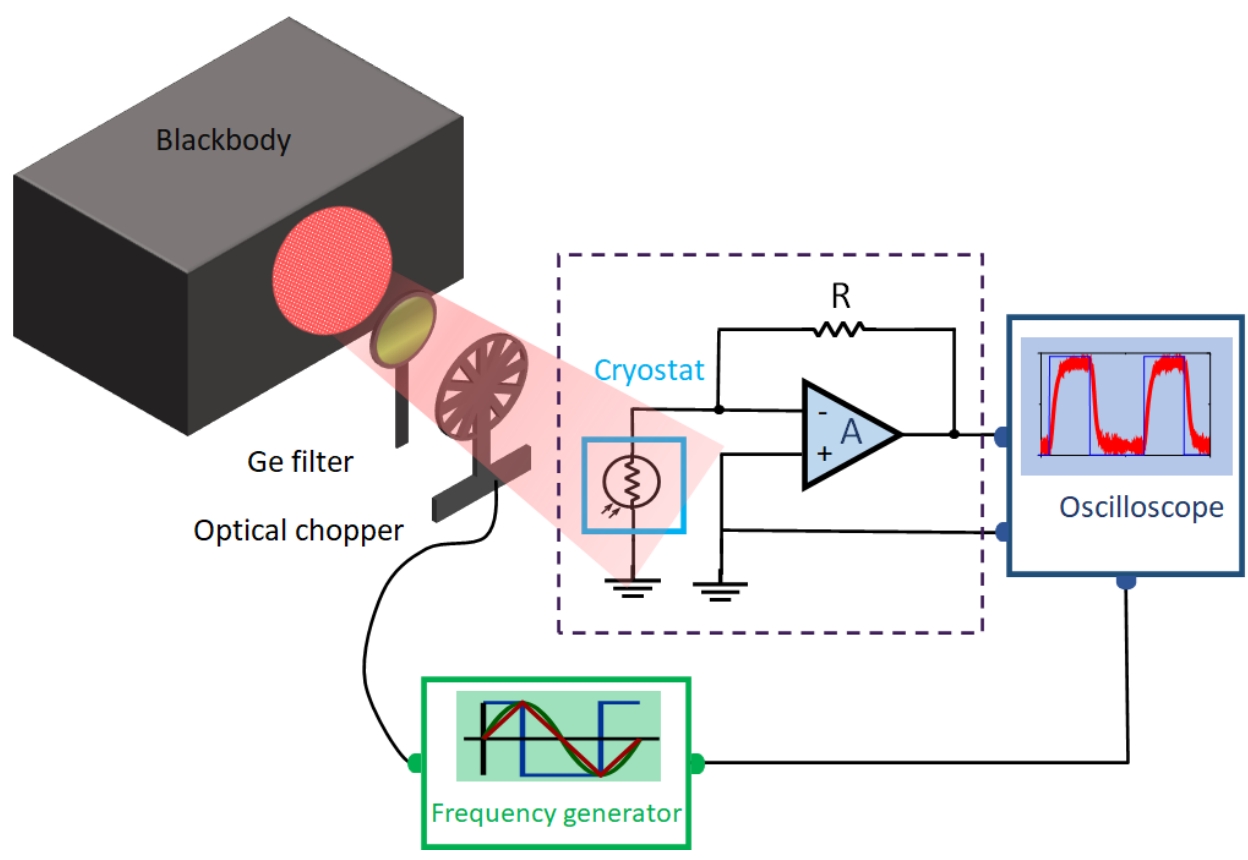

Figure S 11: Photoresponse setup. Set-up for responsivity measurement with the blackbody.

Frequency dependent photocurrent measurement: The photocurrent signal from the sample (inside the cryostat, placed under voltage bias) is amplified with a Femto DLPCA 200 amplifier then fed to an oscilloscope. For irradiation, we use a $1550 \mathrm{~nm}$ laser diode modulated by a frequency generator. Thus, a modulation up to $10 \mathrm{kHz}$ can be obtained. A measurement scheme is shown in Figure S 12. 


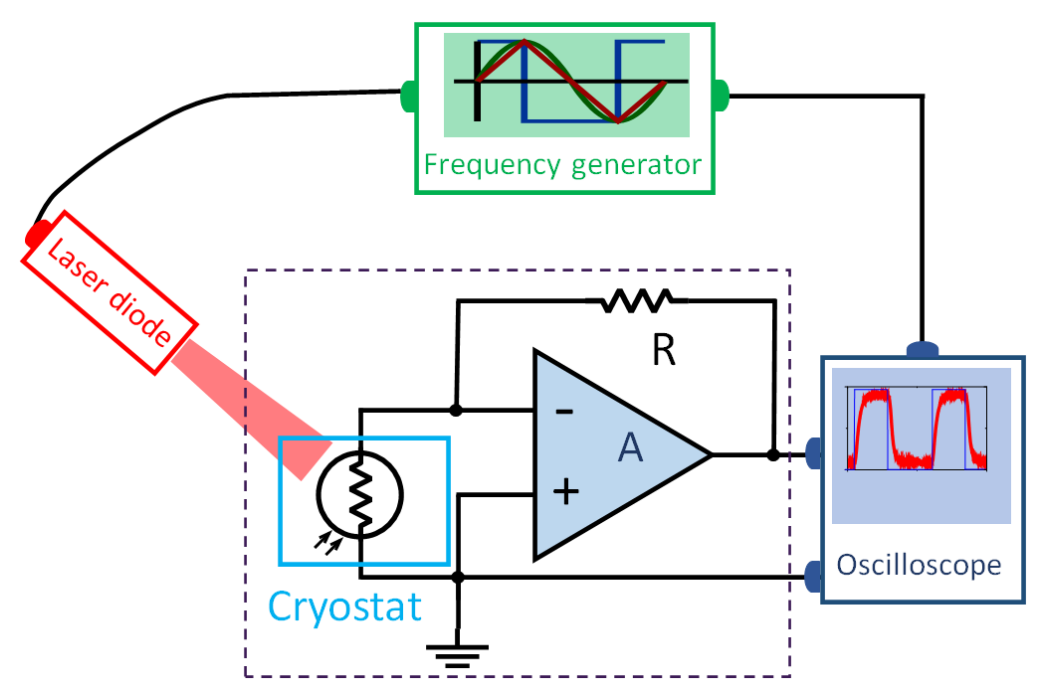

Figure S 12: Time response setup. Scheme diagram of frequency dependent photocurrent measurements.

Noise measurement and detectivity: Figure $S 13$ shows the measured noise current density of our device. The device is biased with a Femto DLPCA 200 amplifier in noise measurements, while the signal is acquired on an SR780 spectrum analyzer. The noise spectra are typically acquired over a frequency range of $3 \mathrm{kHz}$, typically from $10 \mathrm{~Hz}$ to $3000 \mathrm{~Hz}$. For one measurement, the final noise current density spectrum is obtained after averaging 100 scans.

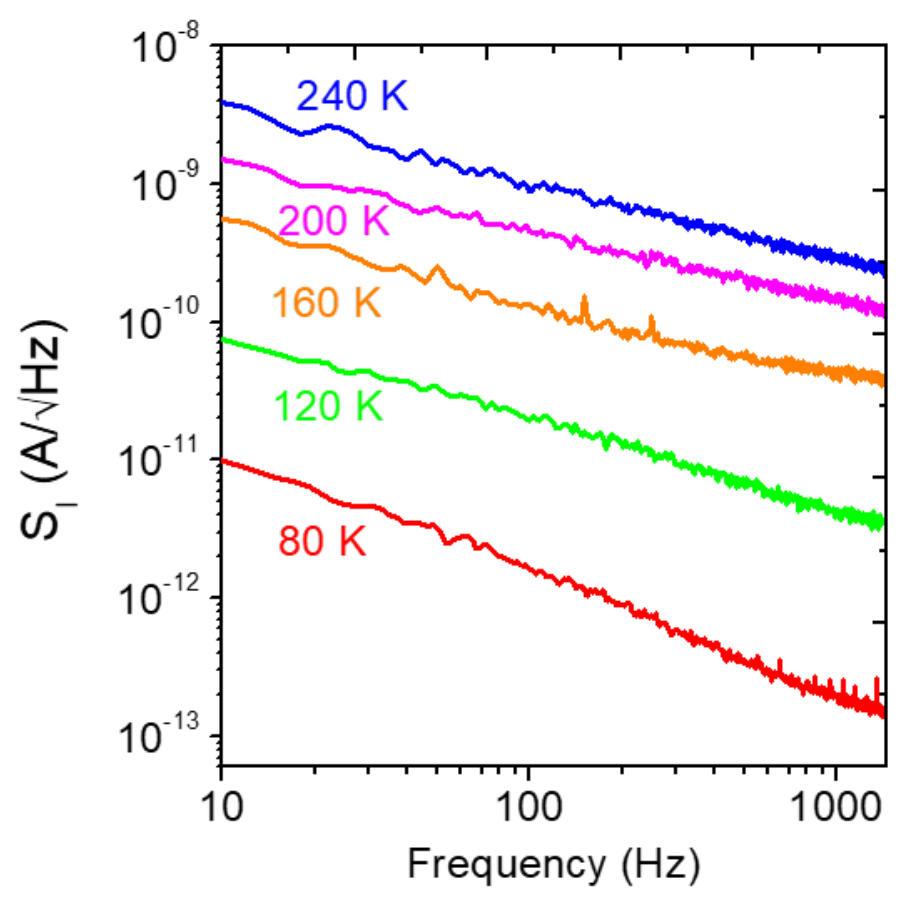

Figure S 13: Noise: Noise current density measured at various temperatures.

Detectivity values are extracted from $D^{*}=\frac{\mathcal{R} \sqrt{A}}{S_{I}}$, where $\mathcal{R}$ is the responsivity of the device, A is the device area (i.e., $3.5 \times 10^{-4} \mathrm{~cm}^{2}$ ), and $S_{I}$ is the measured noise current density. 


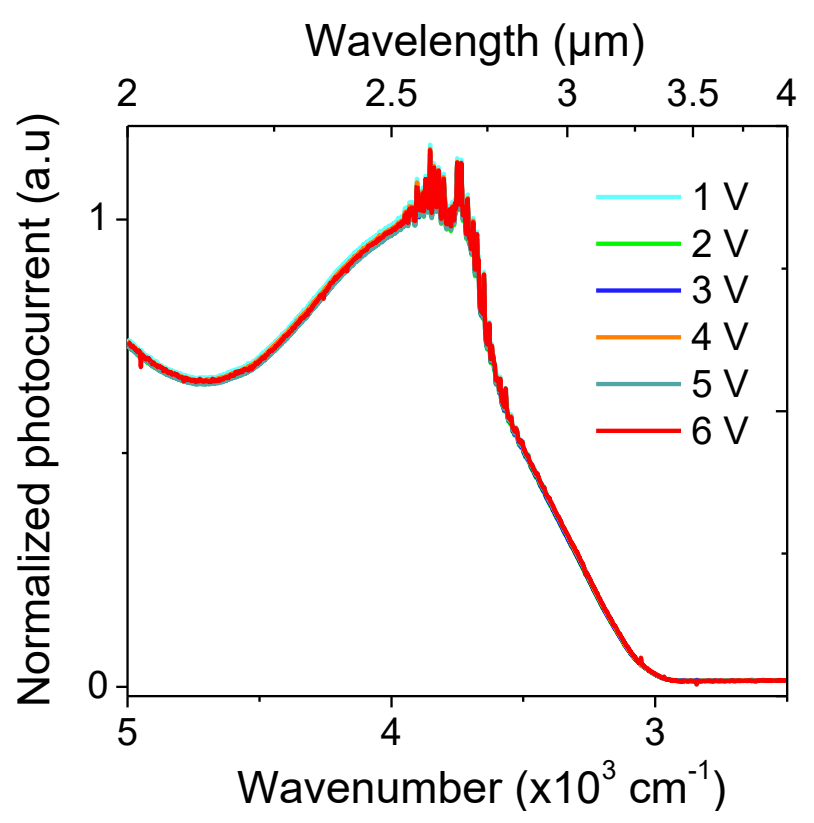

Figure S 14: Effect of bias in the absence of the cavity. Photocurrent spectrum of a NC film on interdigitated electrodes under various bias voltages, at $80 \mathrm{~K}$.

\subsection{Additional electrical characterization}

- Temperature dependence of the dark current

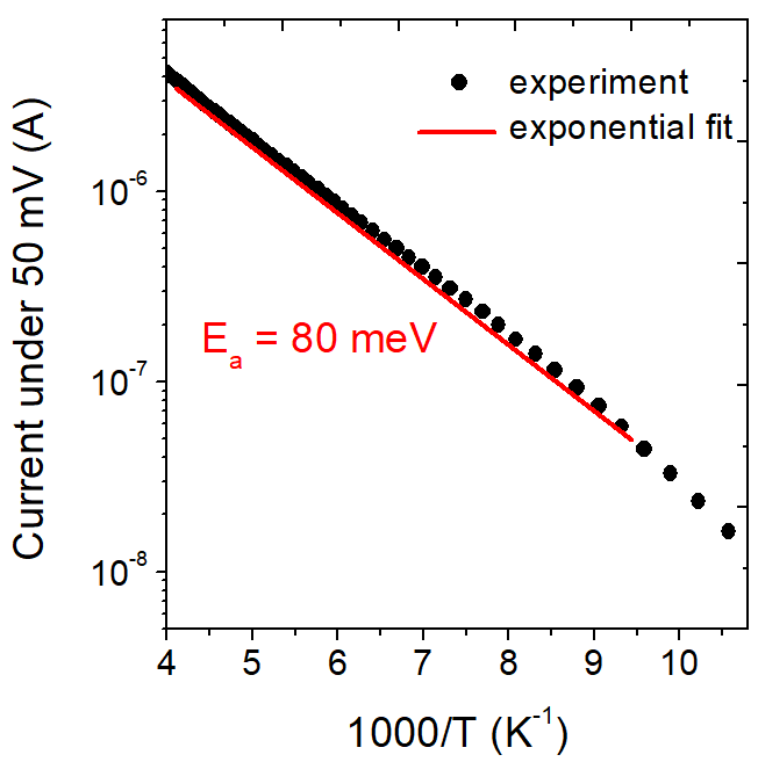

Figure S 15: Temperature dependence of the current. Dark current as a function of the temperature in the cavity device. An activation energy of 80 meV from Arrhenius' fit is also shown.

- Electrolyte gated field-effect transistor (FET):

This measurement is made without illumination. Electrolyte gating is made by mixing $0.5 \mathrm{~g}$ of $\mathrm{LiClO}_{2}$ with $2.3 \mathrm{~g}$ of PEG in a glovebox, then heated at $170^{\circ} \mathrm{C}$ for 2 hours. One drop of electrolyte is brushed 
on top of the HgTe film to make the gate. Then, a copper grid is placed on top of the electrolyte to enhance the uniformity of the electrostatic field due to gating (see Figure $\mathbf{S} 16 \mathrm{a}$ ). The drain-source bias is maintained at $100 \mathrm{mV}$ while the gate bias is swept from $-2 \mathrm{~V}$ to $+2 \mathrm{~V}$ with step of $1 \mathrm{mV}$ by a Keithley 2634b. The measurement is repeated five times.

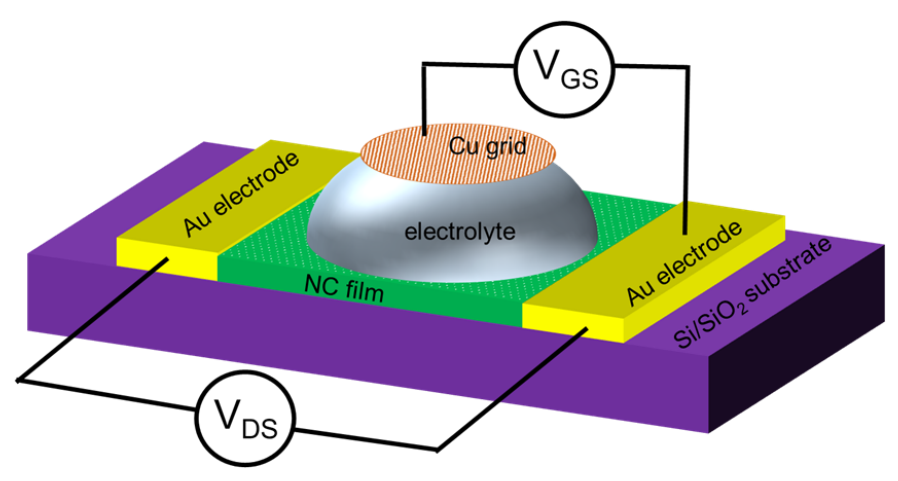

a

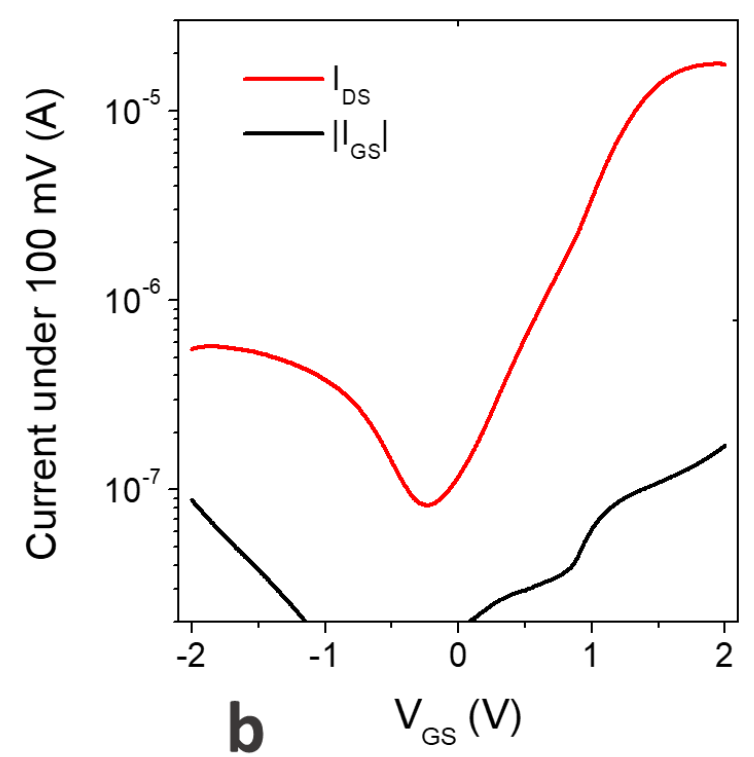

Figure S 16: Field effect transistor measurements. a. Schematic of electrolyte gated field-effect transistor measurement. b. FET transfer curve of the NC film.

\section{- Bias induced shift on TE and TM polarization}

Figure $\mathbf{S} 17$ shows the polarization effect on the device photoresponse, while Figure $\mathbf{S} 18$ shows the effect of bias on the polarized spectra.

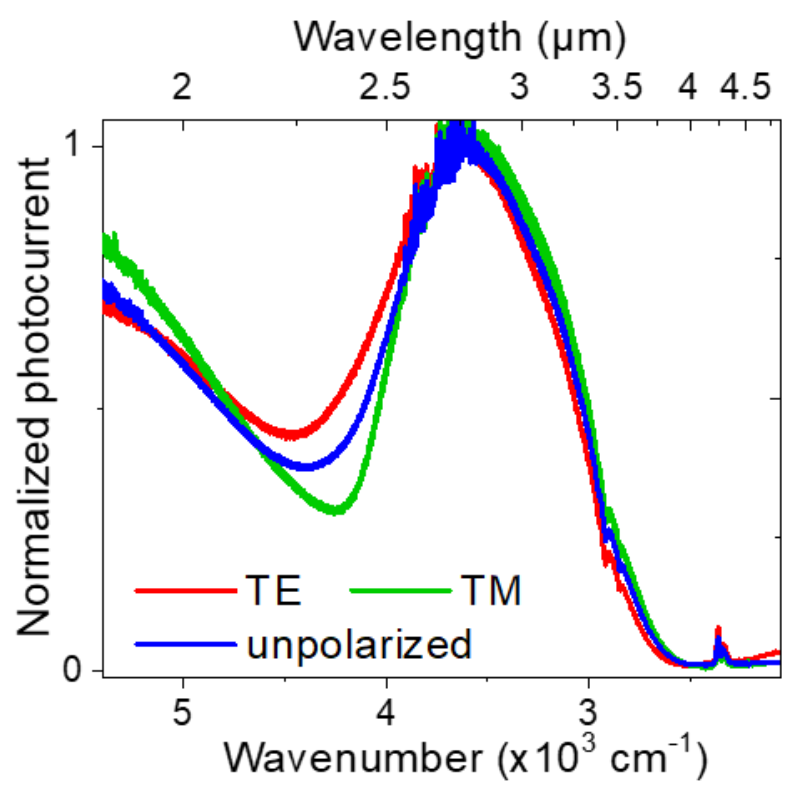

Figure S 17: Effect of polarization of the response. Photocurrent spectra for non-polarized and polarized illumination with the cavity device, at $80 \mathrm{~K}$. 

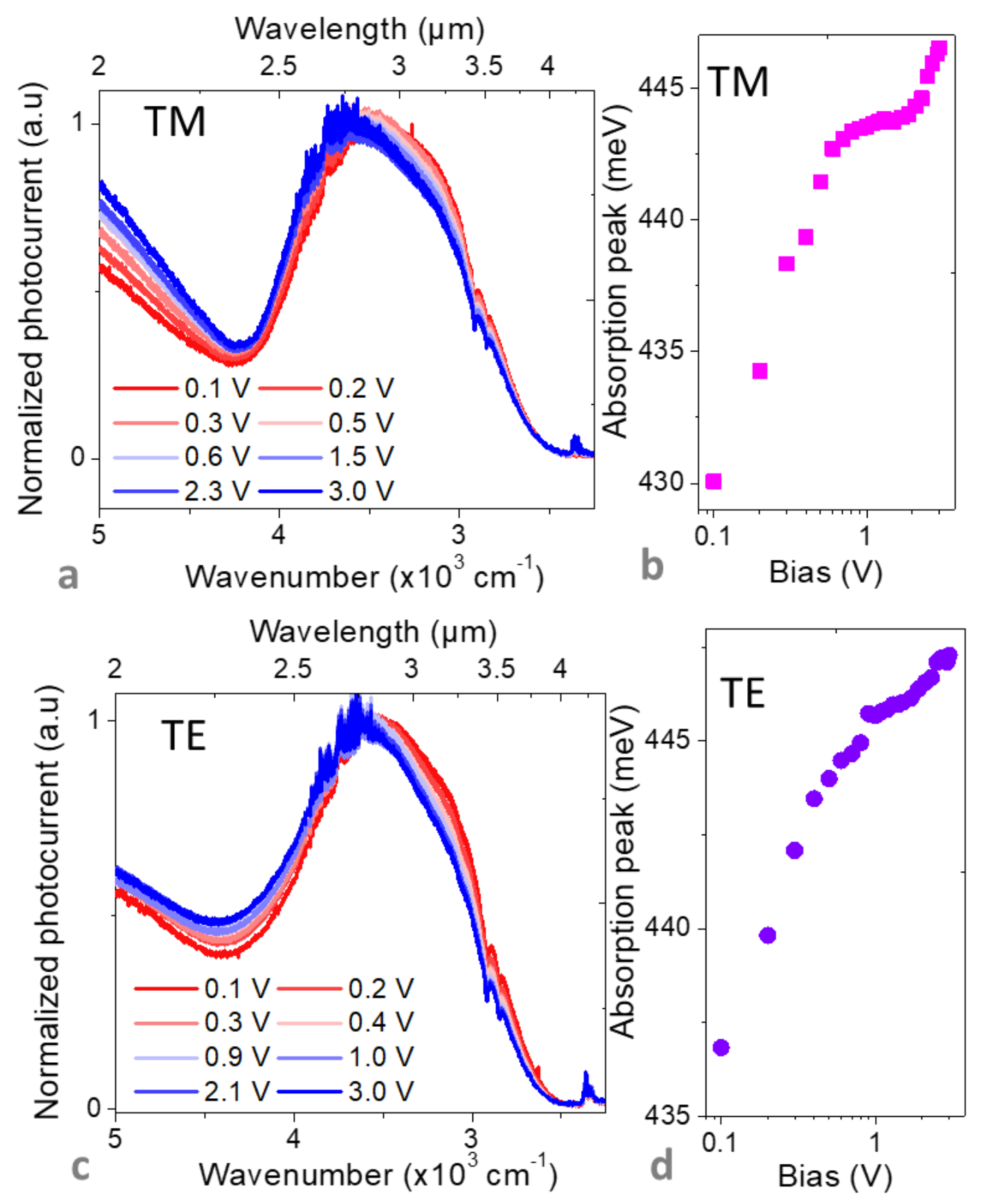

Figure S 18: Effect of polarization on shift. a. (resp. c.) Photocurrent spectra of the cavity device for TM (resp. TE) polarization under various applied bias voltages, at 50 K. b. (resp. d.) Energy of the photocurrent peak as a function of applied bias for TM (resp. TE) polarization. 


\section{- Effect of bias on response}

Note that the sign of the applied bias does not affect the sign of the shift. A blue shift is observed for both positive and negative polarities.
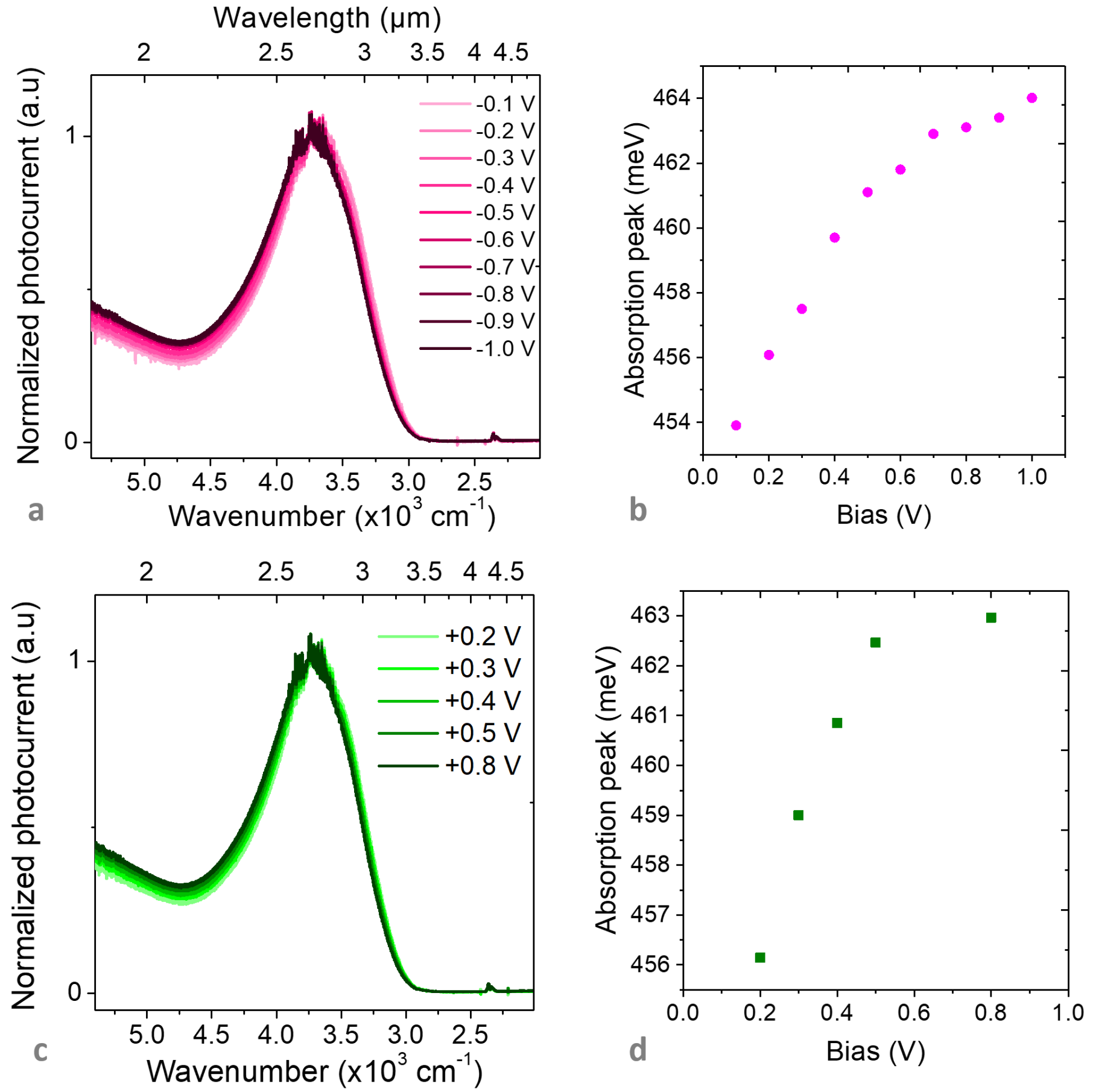

Figure S 19: Effect of bias polarity of the spectral shift. a. Photocurrent spectra for unpolarized light under various negative applied bias voltages. $b$. Energy of the first photocurrent peak as a function of the absolute value of the applied bias. $c$. Photocurrent spectra for unpolarized light under various positive applied bias voltages. $d$. Energy of the first photocurrent peak as a function of the positive applied bias. 
For both cavity and cavityless (i.e., interdigitated electrode) devices, the magnitude of the photocurrent scales quasilinearly with the applied bias, see Figure $\mathbf{S} \mathbf{2 0}$. The current density $(\mathrm{J})$ in our device is given by J=e.n. $\mu$.E where $e$ is the proton charge, $\mathrm{n}$ the carrier density, $\mu$ the carrier mobility, and $E$ the electrostatic field. Under illumination, the carrier density changes from $n$ to $n+\Delta n$ where $\Delta n$ is proportional to the absorption $\alpha$, with $\Delta n=b$. $\alpha$. We can then reformulate $J=e .(n+b \alpha) \cdot \mu$. $E$. Experimentally, we observe a linear scaling (i.e., monotonic increase) of the photocurrent with bias, meaning that the bias dependence of the $b a$ term is very weak.
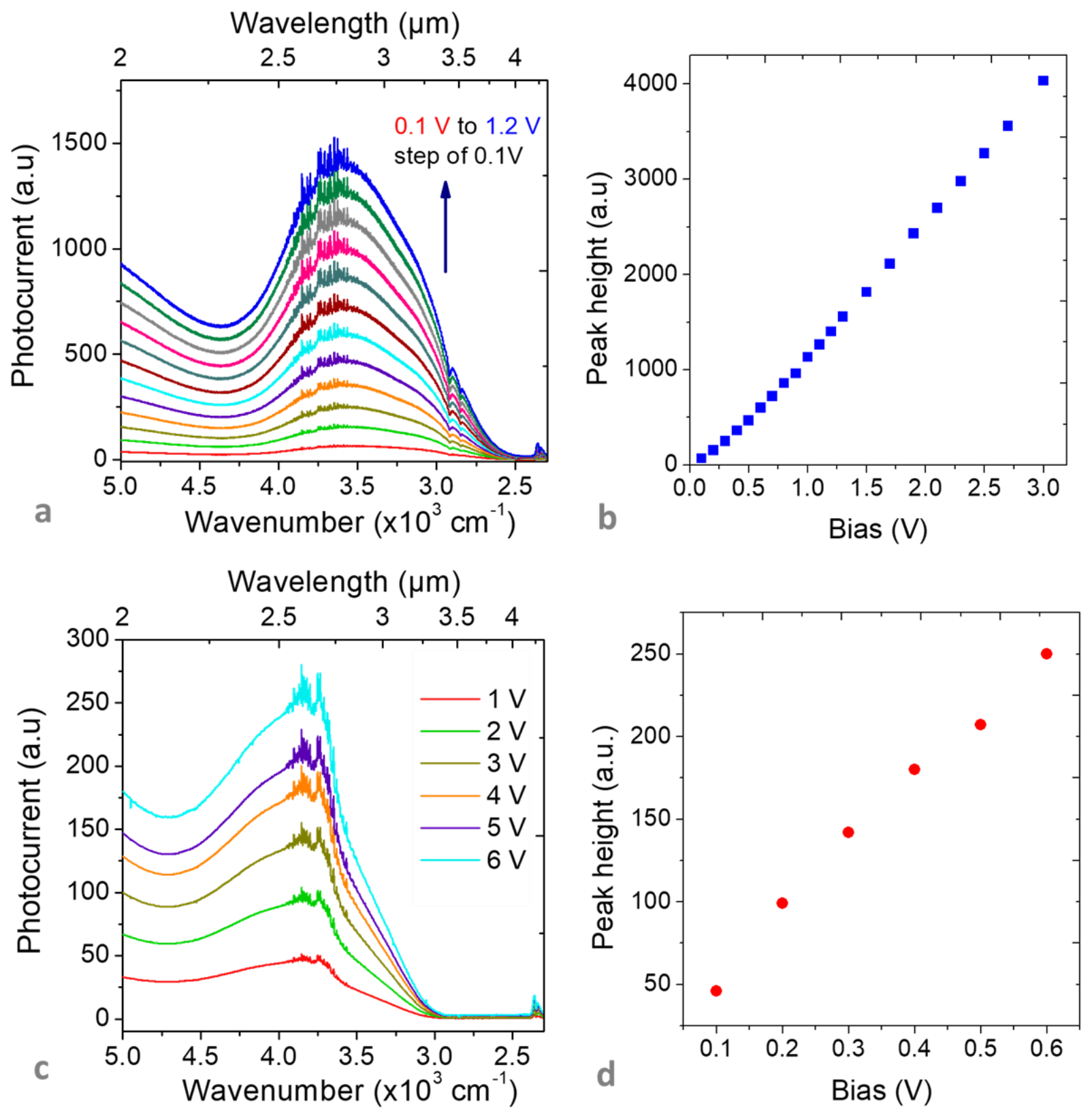

Figure S 20: Photocurrent spectra. a. (resp. b) Photocurrent spectra (resp. photocurrent magnitude) of the cavity device as a function of bias voltage. c. (resp. d) Photocurrent spectra (resp. photocurrent magnitude) of the interdigitated electrode device as a function of bias voltage. 

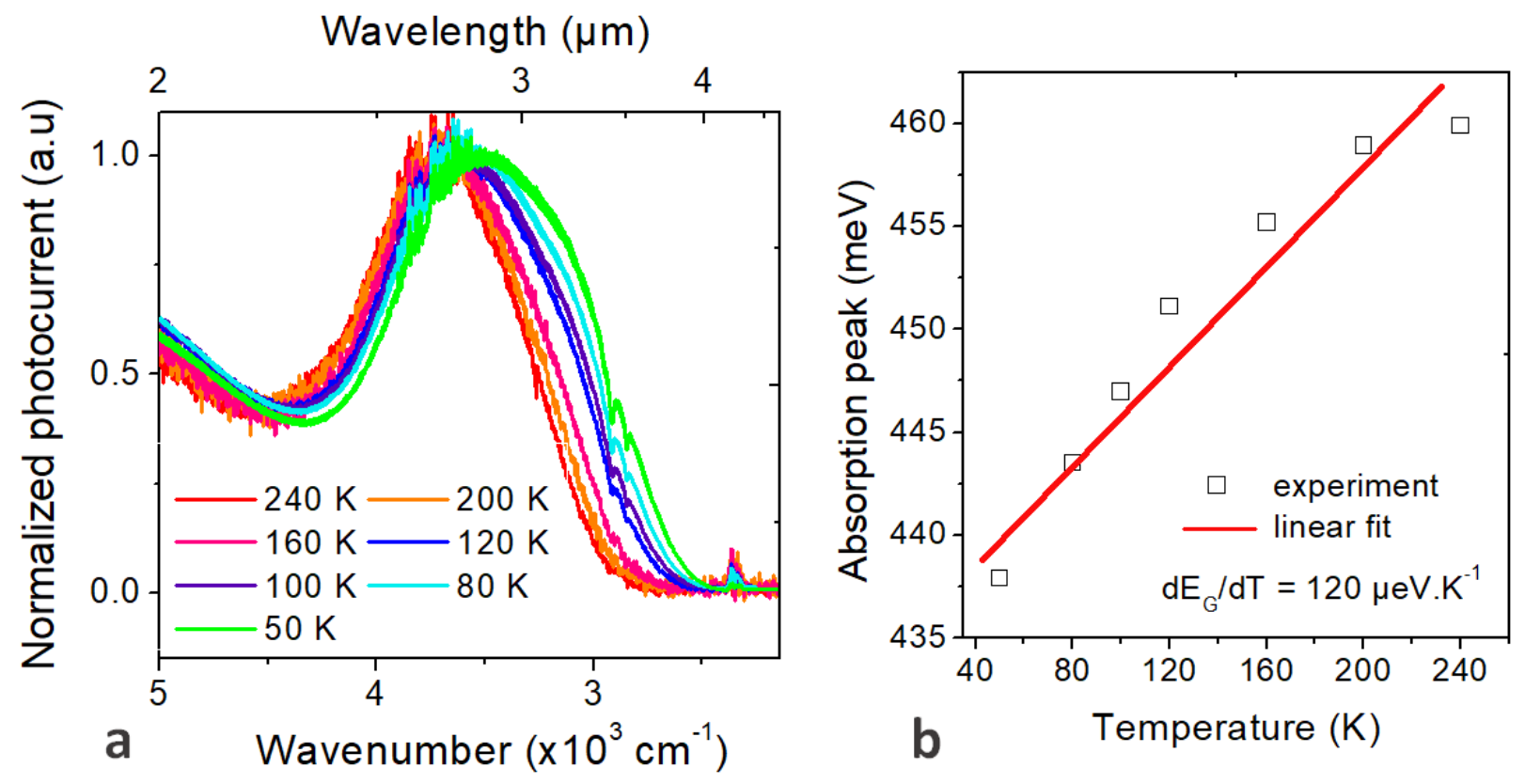

Figure S 21: Temperature-induced spectral shift. a. Unpolarized photocurrent spectra of the cavity device at various temperatures $\left(V_{D S}=0.3 \mathrm{~V}\right)$. b. Energy of the first exciton as a function of the temperature. A linear fit with a $120 \mu \mathrm{eV} . K^{-1}$ slope is also shown.

\section{REFERENCES}

(1) Ordal, M. A.; Bell, R. J.; Alexander, R. W.; Long, L. L.; Querry, M. R. Optical Properties of Au, $\mathrm{Ni}$, and $\mathrm{Pb}$ at Submillimeter Wavelengths. Appl. Opt. 1987, 26, 744.

(2) Rastogi, P.; Chu, A.; Dang, T. H.; Prado, Y.; Gréboval, C.; Qu, J.; Dabard, C.; Khalili, A.; Dandeu, E.; Fix, B.; Xu, X. Z.; Ithurria, S.; Vincent, G.; Gallas, B.; Lhuillier, E. Complex Optical Index of HgTe Nanocrystal Infrared Thin Films and Its Use for Short Wave Infrared Photodiode Design. Adv. Opt. Mater. 2021, 9, 2002066.

(3) Allan, G.; Delerue, C. Tight-Binding Calculations of the Optical Properties of $\mathrm{HgTe}$ Nanocrystals. Phys. Rev. B 2012, 86, 165437.

(4) Moghaddam, N.; Gréboval, C.; Qu, J.; Chu, A.; Rastogi, P.; Livache, C.; Khalili, A.; Xu, X. Z.; Baptiste, B.; Klotz, S.; Fishman, G.; Capitani, F.; Ithurria, S.; Sauvage, S.; Lhuillier, E. The Strong Confinement Regime in HgTe Two-Dimensional Nanoplatelets. J. Phys. Chem. C 2020, $124,23460-23468$. 